\title{
Simultaneous Observations of Atmospheric Electric Field, Aerosols, and Clouds on the R/V Hakuho Maru over the Pacific Ocean
}

\author{
Masashi Kamogawa ${ }^{1^{*}}$, Kosei Ohhora ${ }^{1}$, Sayako Ueda ${ }^{2}$, Kazuhiko Miura ${ }^{2}$, Kazuaki Yajima ${ }^{3}$, \\ Satoshi Hashimoto ${ }^{1}$, Maho Nakamura 1, Yoshihiro Kakinami ${ }^{4}$, Hiroshi Furutani 5, and Mitsuo \\ Uematsu 5
}

1. Department of Physics, Tokyo Gakugei University, Koganei-shi, 184-8501, Tokyo, Japan

2. Department of Physics, Faculty of Science, Tokyo University of Science 1-3 Kagurazaka, Shinjuku-ku, 162-8601, Tokyo, Japan 3. National Institute of Radiological Sciences, 4-9-1 Anagawa Inage, 263-8555, Chiba, Japan

4. School of Systems Engineering, Kochi University of Technology, Miyanokuchi 185, Tosayamada, Kami, Kochi 782-8502, Japan 5. Atmosphere and Ocean Research Institute, The University of Tokyo 5-1-5 Kashiwanoha, Kashiwa, 277-8564, Chiba, Japan

* Corresponding to Masashi Kamogawa (kamogawa@u-gakugei.ac.jp).

\begin{abstract}
There is an ionospheric potential between conductive solid-earth and ionosphere which reaches approximately $250 \mathrm{kV}$. The ionospheric potential is generated by the spherical shell capacitance of which is formed by positively charged ionosphere and negatively charged solid-earth. The capacitance is charged and discharged by the global thunderstorm activity and air-earth current in the fair weather, respectively. This large-scale electric circuit is termed a global electric circuit. Recently, it is pointed out that the variation of ionospheric potential is associated with global climate change, so that some of scientists started revisiting this traditional topic. In order to promote their resurvey, we show that a ground-based measurement of atmospheric electric field highly affected by atmospheric clouds, aerosols, and so on is still a useful tool to measure the variation of ionospheric potential through the simultaneous observations of ground-based atmospheric electric field, aerosols, and clouds on the R/V Hakuho Maru over the Pacific Ocean. In the period we obtained Carnegie curve, the observed AEF did not correlate to atmospheric aerosol concentration. The most plausible interpretation is that the observed variation of $\mathrm{AEF}$ reflected the variation of ionosphere potential.
\end{abstract}

Keywords: Atmospheric electricity, Global electrical circuit, Ionospheric potential

\section{Introduction}

In the atmosphere, there is an electric field that is perpendicular to the ground. The electric potential of the ionosphere formed by the atmospheric electric field (AEF) is approximately $250 \mathrm{kV}$ relative to the ground surface (MacGorman and Rust, 1998). The earth is regarded as a spherical shell capacitor, in which the ionosphere is charged positively, whereas a ground is charged negatively. The capacitor discharges constantly through ions produced by cosmic rays and energetic radiation from terrestrial radioactive materials, while it is charged continuously due to global cloud-to-ground lightning activities and precipitations (MacGorman and Rust, 1998).

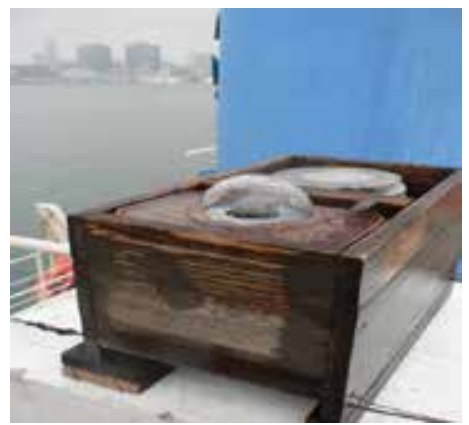

Fig. 1 All sky camera (front) and field mill (behind) set up on on the upper deck of the R/V Hakuho Maru. 
This electric circuit is termed a global electric circuit (GEC), of which a concept was constructed by Thomson and C. T. R. Wilson (Wilson, 1921). A variation of the $\mathrm{AEF}$ is considered to shows the same feature all over the world. A diurnal variation of the $\mathrm{AEF}$ is named Carnegie curve (see MacGorman and Rust (1998)). In a sense, Carnegie curve means that the diurnal variation of the $A E F$ is caused by that of total charges of cloud-to-ground lightning and the precipitations. The AEF, aerosols and ions are closely related with each other (Harrsion and Carslaw, 2003). Positively and negatively charged small ions become the main carriers of the air-earth current because of the large mobility in comparison with charged aerosols. In general, the air-earth current shows a few pico-ampere per square meters. There exists nano-meter-sized and micro-meter-sized aerosols in the lower atmosphere and they adsorb positively and negatively charged ions.

An aerosol particle adsorbs several

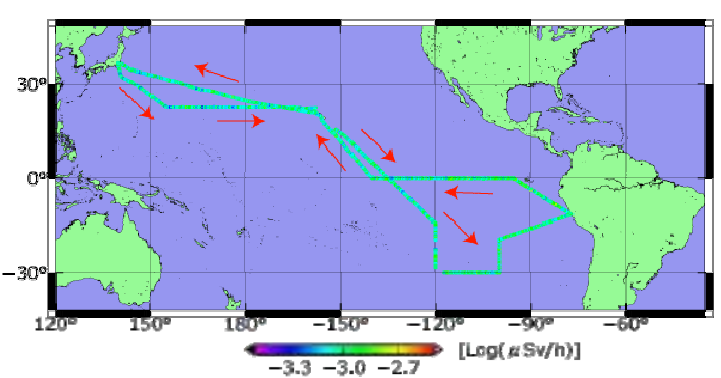

Fig. 2 Ship tracks of KH-11-10 (Tokyo to Peru, December 2011 to January 2012) and KH-12-1 (Peru to Tokyo, January 2012 to March 2012). Arrows represent routes of each cruise. Dose of gamma ray observed on-board is drawn on the routes by the color codes.

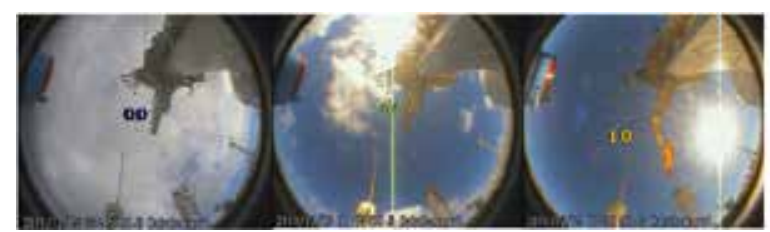

Fig. 3 Examples of sky images observed by all-sky camera. Amount of cloud in the zenith direction was quantified to identify fair weather. Cloudy, semifair weather and fair weather were defined as 0 , 0.5 , and 1.0 . positively and negatively charged ions, resulting in compensation of excess charges. With the aerosol increase, the concentration of ions in the atmosphere decreases and as a result a conductivity o decreases. Since the air-earth current density is considered to be approximately constant on the fair-weather under the less number of aerosols in the large area, a local increase of aerosols is compensated by an increase of AEF $\boldsymbol{E}$ according to the following Ohm's law:

$$
\boldsymbol{j}=\sigma \boldsymbol{E}
$$

An observation of the AEF is very sensitive to the local changes of weather such as not only thunderstorm but also nonthunderstorm cloud (MacGorman and Rust, 1998). In order to measure the AEF variation originating from ionospheric potential changes, the observation on the condition of less aerosols and high rate of fair weather days should be carried out, which means that the observation on the ship such as Carnegie research vessel (see MacGorman and Rust, 1998) is suitable. Thus, in this study, we first follow the Carnegie-type observation and try to simultaneously measure various

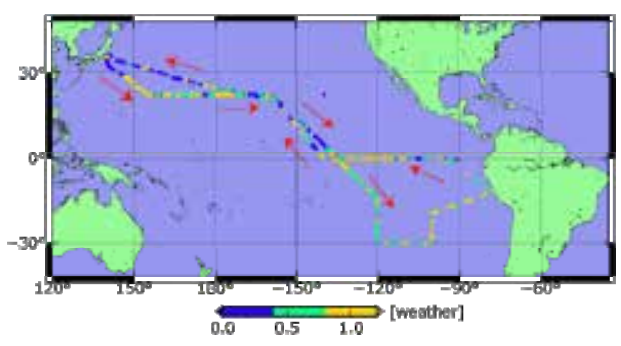

Fig. 4. Amount of cloud defined in Fig. 3 is drawn on the routes by the three color codes along the ship tracks during KH-11-10 (Tokyo to Peru) and KH-12-1 (Peru to Tokyo). Arrows represent routes of each cruise 
parameters related to AEF including aerosols to discuss possibility that variation of AEF reflects the variation of ionosphere potential or not.

\section{Measurement}

Electric resistance of the air near the ground surface is very high, and the AEF over the ground surface cannot be measured by means of a simple voltage checker, because an inner electric resistance of the checker is much smaller than that of the air. A detection instrument of the AEF called a field mill has been frequently used (MacGorman and Rust, 1998). The field mill records the AEF (12 bit resolution and $2 \mathrm{~Hz}$ sampling) which periodically induces the charges on the exposure metallic plate with rotating a

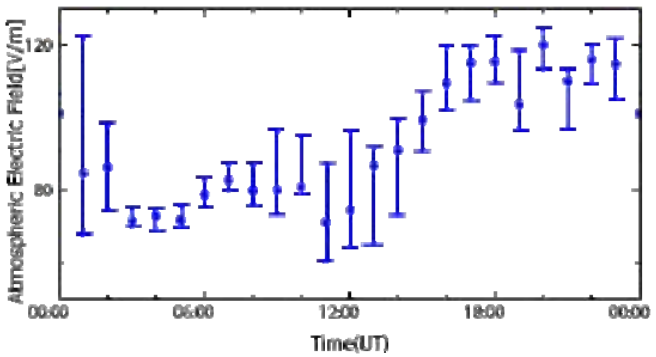

Fig. 5 Diurnal variation of the observed atmospheric electric field on-board R/V Hakuho Maru in universal time (UT) Error bars is 5\% and $15 \%$ percentiles. metallic-shielding chopper. In this study, we used a commercial product which is made in Canada (EMF-100, Boltek Co. Ltd.).

The field mill was installed on the $\mathrm{R} / \mathrm{V}$ Hakuho Maru during a trans-Pacific research cruise, which encompassed the North, South, and equotiral Pacific Oceans between Japan and Peru to measure the AEF in fair weather (Fig. 1). This project has been performed from December, 2011 to the beginning of March, 2012. An amount of cloud in the zenith direction was observed simultaneously in order to compare with the AEF by using all sky-camera every 15 minutes. Moreover, the AEF was observed on the beach near the Shibaura wharf in the Tokyo Port just after arrival of the course in order to calibrate the AEF measured at the R/V Hakuho Maru on 7 March, 2012.

Size segregated aerosol number concentration was observed with optical particle counters (OPC) of Rion Co. Ltd. (KC18). The measurable diameter of this OPC is above $0.1 \mu \mathrm{m}$. A particle

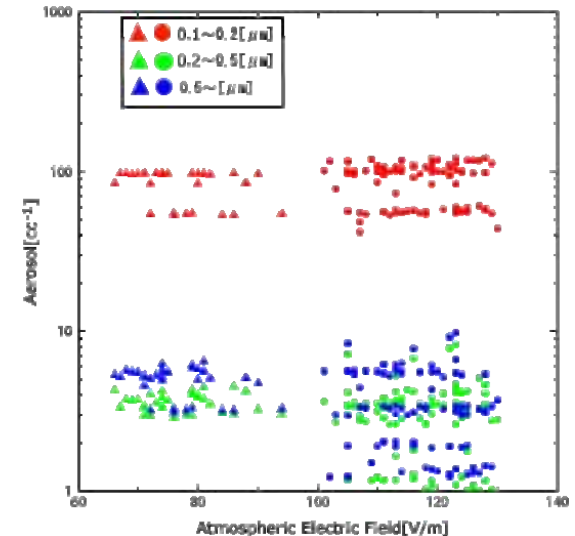

Fig. 6 Relationship between the intensity of atmospheric electric field and the number of aerosol. Color indicates size of aerosol. Solid circles and triangles denote the period from 03:00 to10:00 and from 16:00 to 24:00 as shown in Fig. 5. number concentration in each diameter range is estimated by means of the measurement of the Mie scattering of light. A system was set in a container situated on the front of upper deck. Observed air was taken through the inlet attached on a front roof of the container. The integrated numbers of each diameter every 1 minute were measured by OPC after drying the air by diffusion dryer. Humidity of the dry air is lower than $30 \%$. Data affected by the exhaust gas of the vessel engine were excluded.

In addition, an ionizationchamger-type survey meter (AE-233L, APPLIED ENGINEERING Inc.) was set up in the first laboratory inside the R/V Hakuho Maru and gamma-ray was observed. A measurement was done every 20 seconds and recorded as an averaged dose every 10 minutes. The instrument installed inside the vessel detects not only cosmic ray but also gamma ray originating from radioactive materials embedded in the ship apparatuses. However, the latter is not expected to vary, because the apparatuses and materials are fixed tightly on 
the vessel.

The trans-Pacific voyage by the $\mathrm{R} / \mathrm{V}$ Hakuho Maru was classified into two cruises: One is $\mathrm{KH}-11-10$ voyage from Tokyo to Peru, of which the main observations is poor nutritious area in the off-Peru, while the other is $\mathrm{KH}-12-1$ from Peru to Tokyo, of which the main observations is rich nutritious area in the equatorial region. The KH-12-1 cruise was named as Equatorial Pacific Ocean and Stratospheric/Tropospheric

Atmosphere Study (EqPOS) since unique activity on atmospheric stratospheric and tropospheric observation were also carried out during the cruise.

\section{Results}

Small ions, the major charge carriers of air-earth current, are produced by cosmic rays and terrestrial radioactive materials such as radon gas from ground surface. Since radon is very soluble to water, in the voyage the concentration variation of small ions associated with the gamma rays is almost all originated from cosmic rays (Kowatari et al., 2012). From Fig. 2, it is obvious that the variation of the gamma rays is very small for the whole voyage period. As already shown by Kowatari et al. (2012), the latitude
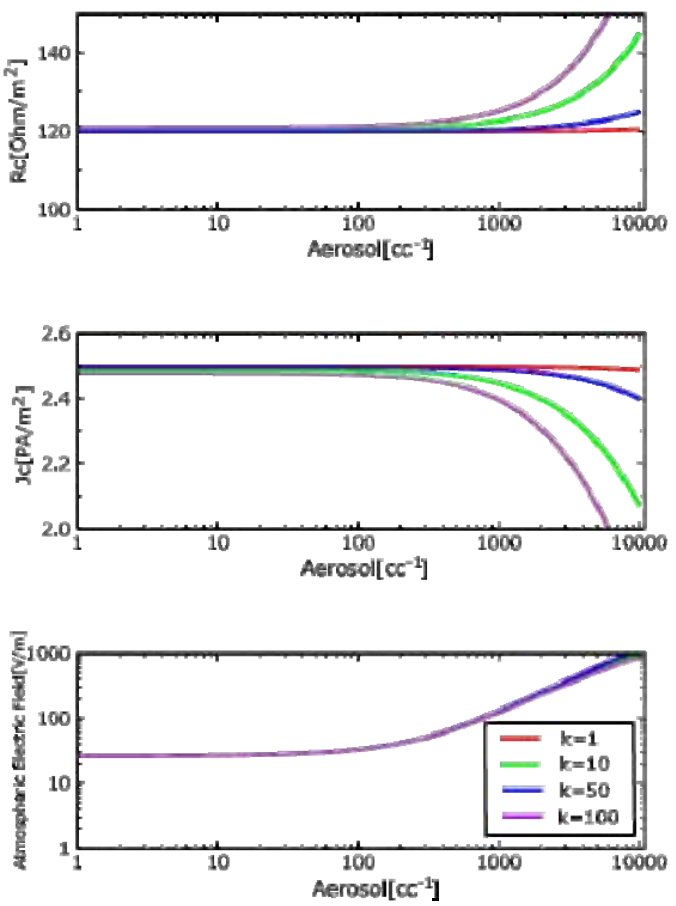

Fig. 7 Calculated data of total columnar resistance, air-earth current, and atmospheric electric field on the ground versus aerosol containing from the ground to altitude $k[\mathrm{~m}]$. dependence of the dose rate near the equator is hardly observed. Therefore, the variation of ions produced by gamma rays is very small, and the number of cosmic-ray-origin small ions is almost constant throughout the voyage

To identify the fair weather, the amount of clouds is qualitatively classified into three steps $(0,0.5$, and 1.0 ) as shown in Fig. 3. The fair weather frequently used in GEC study is equal to 1.0. Figure 4 shows the amount of cloud for all over the voyage. From the result shown in Fig. 4, we focused on our analysis to off-Peru, since the amount of cloud was relatively small for long observation period. The diurnal variation of AEF during this period is shown in Fig. 5. It should be noticed that the curve shows a peak at about 1900 in universal time (UT) and very resembles to Carnegie Curve. Figure 6 shows a correlation between the AEF and the concentration of aerosols. Here, the AEF data is used only within the error bars shown in Fig. 5. In Fig. 6 triangles and circles illustrate the time durations of $0300-1000$ and $1600-2400$, respectively. From equation (1), the AEF increases with increasing the number of aerosols. However, positive correlation between the AEF and the concentration of aerosols was not seen.

\section{Numerical verification}

In order to interpret the result shown in Fig. 6, we follow the numerical analysis employed by Harrison et al. [2009]. The following equation (2) denotes the concentration variation of small ions in the left side, the production of ions due to cosmic rays and terrestrial radioactive materials in the first term of right side, annihilation due to recombination and attachment to the aerosol particles. Accordingly, balance equation (2) of ions is given by

$$
\frac{d n}{d t}=q-\alpha n^{2}-\beta n N
$$

where, $n$ and $N$ are concentration of ions and aerosols, and $q, \alpha, B$ represent ion pair production 
rate and the coefficients of small ion recombination and attachment of small ions to aerosols, respectively (Harrsion and Carslaw, 2003). Coefficient of attachment of small ions to aerosol is given by an inverse of the life time of the small ion. Since in the steady state the term in the left side $d n / d q$ is neglected, atmospheric conductivity in the layer near the ground surface is written by

$$
\sigma_{s}=2 n \mu e=\mu e \frac{\left[-\beta \mathrm{N}+\sqrt{\beta^{2} N^{2}+4 \alpha q}\right]}{\alpha},
$$

where $n$ is the concentration of small ion, $\mu$, the mobility of small ion, is $1.2 \times 10^{-4} \mathrm{~m}^{2} \mathrm{~V}^{-1} \mathrm{~s}^{-1}, \alpha$ is recombination coefficient of small ions, is $1.6 \times 10^{-12} \mathrm{~m}^{3} \mathrm{~s}^{-1}, e$ is the charge of electron, $N$ is the concentration of aerosol, and $B$ is the attachment coefficient of small ion to aerosol. Attachment coefficient is about $4 \times 10^{-11} \mathrm{~m}^{3} \mathrm{~s}^{-1}$ in the aerosol with the average radius of $0.17 \mu \mathrm{m}$ at off-Peru, following the paper by Harrsion and Carslaw (2003). Variation of ion mobility is assumed to be constant. Ion pair production rate is $10^{7} \mathrm{~m}^{-3} \mathrm{~s}^{-1}$ which is general value at the surface of the continent (Harrison et al 2008). Air-earth electric current is subject to ionosphere potential $V_{i}$ and electric resistance of columnar perpendicular to the surface. It is known that in the atmosphere the resistance of the columnar per unit area $R_{\mathrm{C}}$ is $100-170 \Omega / \mathrm{m}^{2}$. The major columnar resistance is given by the resistance $R_{\mathrm{BL}}$ of the boundary layer up $2 \mathrm{~km}$ altitude. The other resistance $R_{\mathrm{FT}}$ is given by the resistance at free troposphere, which is over the boundary layer. Harrison and Bennett (2007) proposed the following linear approximation when the disturbance at the boundary layer is used like

$$
R_{\mathrm{C}}=k / \sigma_{\mathrm{S}}+R_{\mathrm{FT}}
$$

where $\sigma_{s}$ is the conductivity of surface atmosphere having altitude $k$. Air-earth current $J_{\mathrm{C}}$ is given by equation (5) by using ionosphere potential $V_{\mathrm{i}}$ and Ohm's law.

$$
J_{C}=V i / R c=V i /\left[k / \sigma_{s}+R_{\mathrm{FT}}\right]
$$

In the present study, variation of atmospheric electric field is numerically evaluated in the case of uniform appearance of the aerosols in the altitude with equations (1)-(5). As a result, in the case of the averaged aerosol concentration measured off-Peru area is about 100 from Fig. 6 . From Fig. 7, in this concentration range AEF does not change even if the aerosols were distributed up to $500 \mathrm{~m}$ in altitude. AEF near the surface is enhanced, when several thousand aerosols is produced. According to the present result shown in Fig. 6, the variation of aerosol is also constant and not correlated to the variation of AEF because the production rate of ions is constant. Therefore, measured variation of AEF is mostly expected to be attributed to the variation of ionosphere potential

In general, ionosphere potential has been observed by the use of balloon, rocket and airplane. However, time resolution of the measurements is not good. Therefore observation over the sea shows a promising method to monitor the ionosphere potential under the condition of low concentration aerosols and fair weather. This measurement may contribute to the study of ionospheric potential change associated with climate changes.

\section{Conclusion}

$\mathrm{AEF}$ and electric conductivity were evaluated throughout the $\mathrm{KH}-11-10$ and $\mathrm{KH}-12-1$ Hakuho Maru cruises. In the period we obtained Carnegie curve, the observed AEF did not correlate to atmospheric aerosol concentration. The most plausible interpretation is that the observed variation of AEF reflected the variation of ionosphere potential. Recently an attention has been paid to close relation between ionosphere potential and climate change. Present result shows that ground-based AEF observation possibly contributes to monitor the ionosphere potential.

Acknowledgments. We thank Prof. Giles Harrison (Department of Meteorology, University of Reading) for his calculation algorism of ion concentration. Authors also thank the crew of the R/V Hakuho Maru during KH-11-10 and KH-12-1 cruise for their kind assistance on our shipboard observation. This research was partially supported by the Ministry of Education, Science, Sports and Culture, Grant-in-Aid for Young Scientists (B), No. 21710180, 2009 (M. K.). 


\section{References}

Feynman, R., R. Leighton and M. Sands, Electricity in the Atmosphere (Chapter 9), in The Feynman Lectures on Physics (6th. ed.), Vol. II, Addison Wesley Longman, 1977.

Harrison, R. G., K. L. Aplin, M. J. Rycroft, Atmospheric electricity coupling between earthquake regions and the ionosphere, J. Atmos. Solar-Terr. Phys., 72, 376-381, 2009.

Harrison, R. G., and A. J. Bennett, Multi-station synthesis of early twentieth century surface atmospheric electricity measurements for upper tropospheric properties, Adv. Geosci., 13, 17-23.2007

Harrison, R. G., and H. Tammet, Ions in the terrestrial atmosphere and other solar system atmospheres, Space Sci. Rev., 137, 107-118, 2008.

Harrison, R. G., and K. S. Carslaw, Ion-aerosol-cloud processes in the lower atmosphere, Rev. Geophys.,41, 3, 1012-1038, 2003.

Kowatari, M., Y.Ohta, S. Satoh, K. Nagaoka, J. Abukawa and T.Nakamura, Evaluation of Geomagnetic Latitude Dependence of the Cosmic-ray Induced Environmental Neutrons in Japan, Journal of Nuclear Science and Technology, 44, 2, 114-120, 2007.

MacGorman, D. R. and W D. Rust, The Electrical Nature of Storms, Oxford University Press, 422p. 1998.

Nagaoka, K., I. Hiraide, K. Sato and T. Nakamura, Nationwide measurements of cosmic-ray dose rates the throughout Japan, Radiation Protection Dosimetry,132, 4, 365-374, 2009.

Wilson, C. T. R., Investigations on Lightning Discharges and on the Electric Field of Thunderstorms, Philos. Trans. Roy. Soc. Lond. A, 221, 73, 1921.

(Received September 25, 2013; revised January 5, 2014; accepted January 7, 2014) 